\title{
Intervention randomized controlled trials involving wrist and shoulder arthroscopy: a systematic review
}

\author{
Kamelia Tadjerbashi ${ }^{* *}$, Roberto S Rosales ${ }^{2}$ and Isam Atroshi ${ }^{1,3}$
}

\begin{abstract}
Background: Although arthroscopy of upper extremity joints was initially a diagnostic tool, it is increasingly used for therapeutic interventions. Randomized controlled trials (RCTs) are considered the gold standard for assessing treatment efficacy. We aimed to review the literature for intervention RCTs involving wrist and shoulder arthroscopy.
\end{abstract}

Methods: We performed a systematic review for RCTs in which at least one arm was an intervention performed through wrist arthroscopy or shoulder arthroscopy. PubMed and Cochrane Library databases were searched up to December 2012. Two researchers reviewed each article and recorded the condition treated, randomization method, number of randomized participants, time of randomization, outcomes measures, blinding, and description of dropouts and withdrawals. We used the modified Jadad scale that considers the randomization method, blinding, and dropouts/withdrawals; score 0 (lowest quality) to 5 (highest quality). The scores for the wrist and shoulder RCTs were compared with the Mann-Whitney test.

Results: The first references to both wrist and shoulder arthroscopy appeared in the late 1970s. The search found 4 wrist arthroscopy intervention RCTs (Kienböck's disease, dorsal wrist ganglia, volar wrist ganglia, and distal radius fracture; first 3 compared arthroscopic with open surgery). The median number of participants was 45 . The search found 50 shoulder arthroscopy intervention RCTs (rotator cuff tears 22, instability 14, impingement 9, and other conditions 5). Of these, 31 compared different arthroscopic treatments, 12 compared arthroscopic with open treatment, and 7 compared arthroscopic with nonoperative treatment. The median number of participants was 60. The median modified Jadad score for the wrist RCTs was 0.5 (range $0-1$ ) and for the shoulder RCTs 3.0 (range 0-5) $(p=0.012)$.

Conclusion: Despite the increasing use of wrist arthroscopy in the treatment of various wrist disorders the efficacy of arthroscopically performed wrist interventions has been studied in only 4 randomized studies compared to 50 randomized studies of significantly higher quality assessing interventions performed through shoulder arthroscopy.

Keywords: Arthroscopy, Wrist, Shoulder, Randomized trials, Jadad scale, Intervention RCT, Systematic review

\footnotetext{
* Correspondence: kamelia_tadjerbashi@hotmail.com

'Department of Orthopedics, Hässleholm Hospital, Hässleholm SE-28125, Sweden

Full list of author information is available at the end of the article
}

\section{Biomed Central}

(c) 2014 Tadjerbashi et al.; licensee BioMed Central Ltd. This is an Open Access article distributed under the terms of the Creative Commons Attribution License (http://creativecommons.org/licenses/by/2.0), which permits unrestricted use, distribution, and reproduction in any medium, provided the original work is properly credited. The Creative Commons Public Domain Dedication waiver (http://creativecommons.org/publicdomain/zero/1.0/) applies to the data made available in this article, unless otherwise stated. 


\section{Background}

Although arthroscopy of upper extremity joints was initially introduced mainly for diagnostic purposes it is being increasingly used for therapeutic interventions [1]. For example, wrist interventions performed through arthroscopy include, among others, excision of wrist ganglia, treatment of acute fractures and of non-unions, ligament repair and reconstructions, repair or debridement of the triangular fibrocartilage complex, ulnar head resection, partial or total removal of carpal bones, and joint fusions [1,2]. A recent study on musculoskeletal upper extremity ambulatory surgery in the United States estimated that 272,148 rotator cuff repairs, 257,541 shoulder arthroscopies excluding those for cuff repairs, 3686 elbow arthroscopies, and 25,250 wrist arthroscopies were performed in 2006 [3]. Arthroscopic interventions generally require special equipment and substantial surgical training and may thus be associated with higher costs than open procedures [4]. In addition, arthroscopic procedures may be associated with various complications [5]. Arthroscopic interventions may, however, be more costeffective if their efficacy is superior to that of nonarthroscopic treatments or if they have similar efficacy but provide additional benefit, such as quicker recovery or lower morbidity. There is strong agreement that goodquality randomized controlled trials (RCTs) are the gold standard for assessing treatment efficacy and that they provide higher level of evidence than observational studies [6]. We reviewed the literature for intervention RCTs involving wrist arthroscopy, and for comparison, shoulder arthroscopy, hypothesizing that the quality of wrist and shoulder RCTs are similar.

\section{Methods}

We performed a systematic review of the literature for randomized or quasi-randomized clinical trials in which at least one arm was an intervention performed through wrist arthroscopy or shoulder arthroscopy. An experienced researcher searched for articles published up to December 2012 in the databases PubMed and Cochrane Library. The search was conducted in accordance with the Preferred Reporting Items for Systematic Reviews and Meta-Analyses (PRISMA) guidelines [7]. The search strategy was applied to PubMed and optimized for the Cochrane database (Additional file 1). We included all RCTs written in English, Spanish, or German. We omitted conference abstracts. We checked the references of the initially included articles to identify other potentially relevant studies and subjected them to a similar selection process.

Three researchers reviewed the selected articles (each article reviewed by at least two researchers) and recorded the following data: the country where the study was conducted, the condition for which the interventions were done, the randomization method, the number of randomized participants, the time of randomization, the outcomes measures used, blinding, and description of dropouts and withdrawals. When appropriate we grouped the conditions for which the interventions were done into diagnostic categories. As a measure of RCT quality we used the Jadad scale [8] as modified by Gummesson et al. [9]. The scale considers the randomization method, blinding and description of dropouts/withdrawals, yielding a score from 0 (lowest quality) to 5 (highest) [9]. A study that describes an appropriate randomization method (such as computer-generated sequence or a random-number table) is awarded 2 points while a study that does not report the randomization method or reports an inappropriate method (such as order of presentation or medical record number) is not awarded any points. Similarly a study that reports blinding (single or double) using an appropriate method is awarded 2 points while use of an inappropriate blinding method or absence of blinding does not yield any points. The blinding method was considered appropriate if the article specified whom the blinding involved and, depending on the nature of the interventions, possible additional measures to ensure the blinding (for example, stating that blinding involved an assessor and that the surgical area was covered during patient assessment or that identical incisions were used for the different surgical procedures). Description of any dropouts or withdrawals (or a statement that no dropouts/withdrawals occurred) is awarded 1 point. The grading according to the modified Jadad scale was done by two researchers independently and any disagreements were resolved by discussion until consensus was reached.

The median modified Jadad scores were calculated for the wrist and shoulder RCTs and were then compared with the Mann-Whitney test. A p-value of less than 0.05 was considered to indicate statistical significance.

\section{Results and discussion \\ Results}

The Medline search showed that the first publications in which wrist arthroscopy or shoulder arthroscopy were mentioned appeared in the late 1970s.

\section{Wrist arthroscopy}

Of 7 possible RCTs obtained in the search, 3 were excluded because they involved postoperative analgesia, leaving 4 intervention RCTs eligible for inclusion (Figure 1; Additional file 2). The 4 RCTs (Table 1) involved Kienböck's disease (arthroscopic versus open surgery), dorsal wrist ganglia (arthroscopic versus open excision), volar wrist ganglia (arthroscopic versus open excision), and distal radius fracture (arthroscopically- and fluoroscopicallyassisted versus fluoroscopically-assisted reduction, followed 


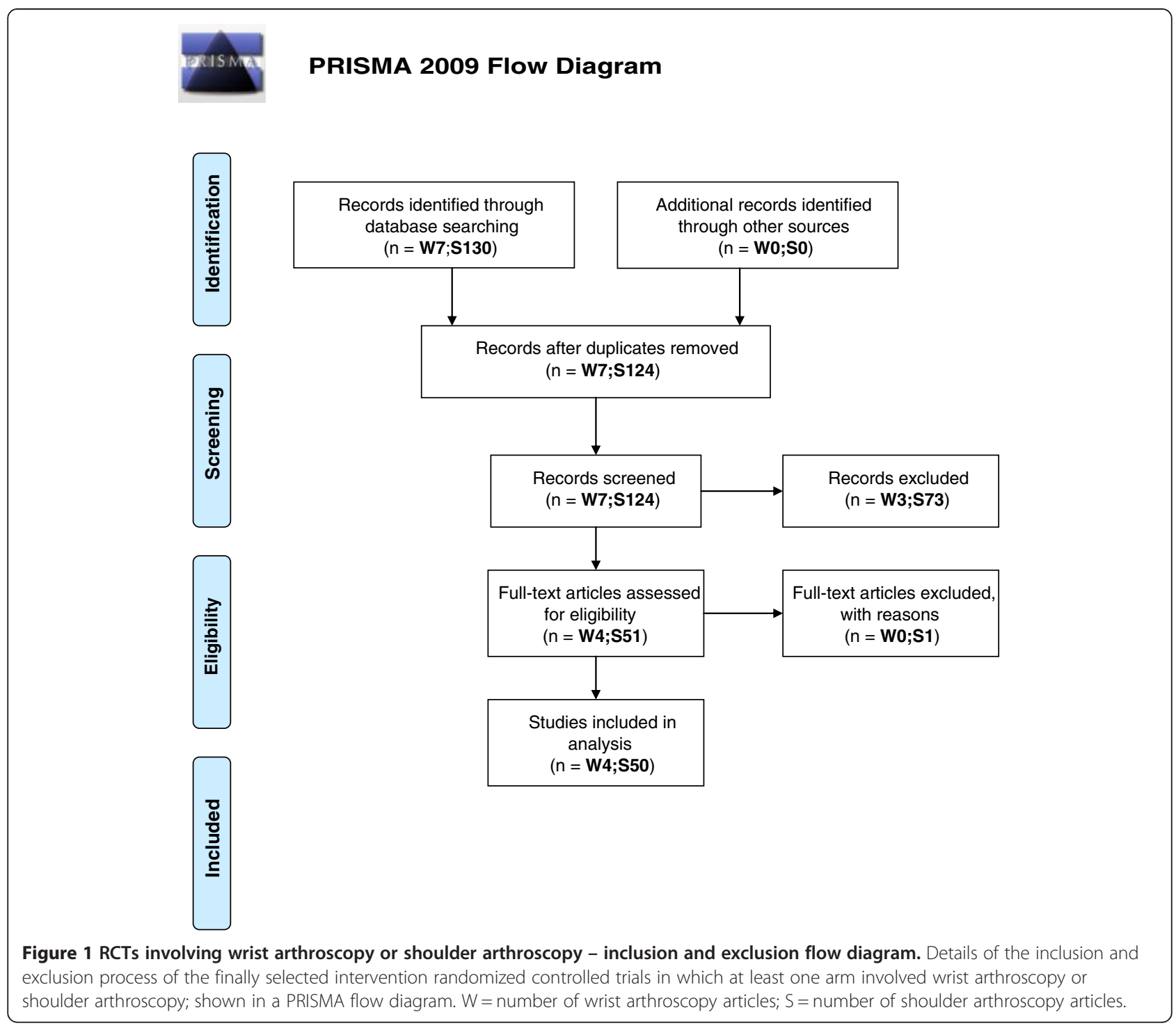

by fixation). The number of participants in the 4 studies was $16,50,72$, and 40 , respectively (median 45 ).

\section{Shoulder arthroscopy}

Of 130 possible RCTs obtained in the search, 80 were excluded: 24 were not intervention RCTs (matched cohort or cross-sectional studies, non-clinical RCTs, RCT protocols), 10 were systematic reviews or meta-analyses, 32 involved anesthesia or postoperative analgesia, 7 involved physiotherapy/postoperative rehabilitation, 6 were subsequent publications of same RCT, and 1 was not intervention through arthroscopy (after review of fulltext and contact with the author). Thus, 50 shoulder intervention RCTs were included (Figure 1; Additional file 2). The $50 \mathrm{RCTs}$ (Table 1 ) involved rotator cuff tears $(\mathrm{n}=22)$, instability $(\mathrm{n}=14)$, impingement $(\mathrm{n}=9)$, and other conditions $(n=5)$. The interventions compared were different arthroscopic procedures $(n=31)$, arthroscopic versus open procedures $(n=12)$, and arthroscopic procedure versus nonoperative treatment $(n=7)$. The median number of participants was 60 (range 17-150).

\section{Trial quality}

Of the 4 wrist studies 2 used inappropriate randomization methods and the remaining 2 stated use of "sealed envelopes" but without reporting how the randomization sequence was generated. None of the studies reported blinding and only 2 provided information about dropouts/ withdrawals. In the 50 shoulder RCTs, the randomization method was described and appropriate in 25 (50\%), described but inappropriate in $18(36 \%)$ and was not described in 7 (14\%). Blinding using an appropriate method was reported in 23 studies (46\%), blinding was reported but the method was inappropriate in $5(10 \%)$ and blinding was not reported in 22 studies (44\%). Dropouts/withdrawals were described in 41 (82\%). 


\begin{tabular}{|c|c|c|c|c|c|c|c|c|c|c|c|c|}
\hline $\begin{array}{l}\text { Author* } \\
\text { (first) } \mathrm{yr}\end{array}$ & Country & Diagnosis & Intervention 1 & N 1 & $\mathrm{D} / \mathrm{W}$ & Intervention 2 & N 2 & W/D & $\begin{array}{l}\text { Randomization } \\
\text { method }\end{array}$ & $\begin{array}{l}\text { Time of } \\
\text { randomization }\end{array}$ & Outcomes & Blinding \\
\hline \multicolumn{13}{|l|}{ Wrist } \\
\hline Kang 2008 & USA & Dorsal ganglion & $\begin{array}{l}\text { Arthroscopic } \\
\text { excision }\end{array}$ & 41 & 13 & Open excision & 31 & 8 & $\begin{array}{l}\text { Medical record } \\
\text { Identifier (odd/ } \\
\text { even) }\end{array}$ & At presentation & $\begin{array}{l}\text { Recurrence, residual } \\
\text { pain, complications }\end{array}$ & NR \\
\hline $\begin{array}{l}\text { Leblebicioglu } \\
2003\end{array}$ & Turkey & Kienböck's disease & $\begin{array}{l}\text { Open } \\
\text { scaphocapitate } \\
\text { fusion and lunate } \\
\text { revascularization }\end{array}$ & 8 & NR & $\begin{array}{l}\text { Arthroscopic scapho- } \\
\text { capitate fusion and capi- } \\
\text { tate pole excision }\end{array}$ & 8 & NR & $\begin{array}{l}\text { Last digit of } \\
\text { Medical record } \\
\text { (odd/even) }\end{array}$ & $N R$ & $\begin{array}{l}\text { Operative time, } \\
\text { LOHS, time to } \\
\text { fusion, ROM, grip, } \\
\text { RTW }\end{array}$ & NR \\
\hline Rocchi 2008 & Italy & Volar ganglion & Open excision & 25 & 2 & Arthroscopic excision & 25 & 1 & $\begin{array}{l}\text { Sealed } \\
\text { envelopes }\end{array}$ & NR & $\begin{array}{l}\text { ROM, grip, scar, } \\
\text { pain, residual } \\
\text { symptoms, } \\
\text { recurrence }\end{array}$ & NR \\
\hline $\begin{array}{l}\text { Varitimidis } \\
2008\end{array}$ & Greece & $\begin{array}{l}\text { Intra-articular distal } \\
\text { radius fracture }\end{array}$ & $\begin{array}{l}\text { Arthroscopic and } \\
\text { fluroscopic assisted } \\
\text { reduction + external } \\
\text { fixation and } \\
\text { percutaneous } \\
\text { pinning }\end{array}$ & 20 & $N R$ & $\begin{array}{l}\text { Fluroscopic assisted } \\
\text { reduction + external } \\
\text { fixation and percutaneous } \\
\text { pinning }\end{array}$ & 20 & $N R$ & $\begin{array}{l}\text { Sealed } \\
\text { envelopes }\end{array}$ & $N R$ & $\begin{array}{l}\text { Mayo wrist score, } \\
\text { DASH (primary), } \\
\text { clinical wrist } \\
\text { instability, grip, } \\
\text { ROM, radiographs }\end{array}$ & NR \\
\hline \multicolumn{13}{|l|}{ Shoulder } \\
\hline $\begin{array}{l}\text { Archetti } \\
\text { Netto } 2012\end{array}$ & Brazil & $\begin{array}{l}\text { Traumatic anterior } \\
\text { instability + } \\
\text { isolated Bankart } \\
\text { lesion }\end{array}$ & Arthroscopic repair & 22 & 5 & Open repair & 28 & 3 & $\begin{array}{l}\text { Computer; } \\
\text { Sealed } \\
\text { envelopes }\end{array}$ & At surgery & $\begin{array}{l}\text { DASH (primary), } \\
\text { UCLA, Rowe, ROM }\end{array}$ & NR \\
\hline Barber 2012 & $\begin{array}{l}\text { USA, } \\
\text { Canada }\end{array}$ & $\begin{array}{l}\text { Large rotator cuff } \\
\text { tear }\end{array}$ & $\begin{array}{l}\text { Arthroscopic single- } \\
\text { row repair + } \\
\text { acellular human } \\
\text { dermal matrix } \\
\text { augmentation }\end{array}$ & 22 & NR & $\begin{array}{l}\text { Arthroscopic single row } \\
\text { repair }\end{array}$ & 20 & NR & $\begin{array}{l}\text { Sealed } \\
\text { envelopes }\end{array}$ & At surgery & $\begin{array}{l}\text { ASES, UCLA, } \\
\text { Constant, MRI, ROM, } \\
\text { strength }\end{array}$ & $\begin{array}{l}\text { Assessor } \\
\text { (radiologist) }\end{array}$ \\
\hline Berth 2010 & Germany & $\begin{array}{l}\text { Massive rotator } \\
\text { cuff tear }\end{array}$ & $\begin{array}{l}\text { Arthroscopic partial } \\
\text { rotator cuff repair }\end{array}$ & 21 & NR & $\begin{array}{l}\text { Arthroscopic debride- } \\
\text { ment + subacromial } \\
\text { decompression }\end{array}$ & 21 & $N R$ & Patient's option & $N R$ & $\begin{array}{l}\text { Constant, ROM, } \\
\text { pain, DASH, } \\
\text { ultrasound }\end{array}$ & NR \\
\hline Bottoni 2002 & USA & $\begin{array}{l}\text { Acute, traumatic, } \\
\text { first-time shoulder } \\
\text { dislocations in } \\
\text { young athletes }\end{array}$ & $\begin{array}{l}\text { Arthroscopic } \\
\text { stabilization }\end{array}$ & 10 & 1 & $\begin{array}{l}\text { Nonoperative treatment ( } 4 \\
\text { wks immobilization } \\
\text { followed by supervised } \\
\text { rehabilitation program) }\end{array}$ & 14 & 2 & $\begin{array}{l}\text { Last digit social } \\
\text { security number } \\
\text { (odd/even) }\end{array}$ & NR & $\begin{array}{l}\text { Recurrent instability, } \\
\text { SANE, L'Insalata } \\
\text { shoulder evaluation, } \\
\text { satisfaction }\end{array}$ & NR \\
\hline Bottoni 2006 & USA & $\begin{array}{l}\text { Recurrent anterior } \\
\text { shoulder instability }\end{array}$ & $\begin{array}{l}\text { Arthroscopic } \\
\text { stabilization }\end{array}$ & 32 & 0 & Open stabilization & 32 & 3 & $\begin{array}{l}\text { Sealed } \\
\text { envelopes }\end{array}$ & $N R$ & $\begin{array}{l}\text { ROM, stability, } \\
\text { SANE, SST, wOSI, } \\
\text { UCLA, Rowe }\end{array}$ & $\begin{array}{l}\text { Assessor } \\
\text { (physiotherapist) }\end{array}$ \\
\hline Brox 1993 & Norway & $\begin{array}{l}\text { Impingement } \\
\text { syndrome (stage II) }\end{array}$ & $\begin{array}{l}\text { Arthroscopic } \\
\text { acromioplasty }\end{array}$ & 45 & 13 & $\begin{array}{l}\text { Supervised exercises; } \\
\text { Placebo laser }\end{array}$ & $50 ; 30$ & $8 ; 4$ & NR & $\begin{array}{l}\text { Mean } 2 \\
\text { months before } \\
\text { treatment }\end{array}$ & $\begin{array}{l}\text { Neer shoulder score } \\
\text { (primary), pain }\end{array}$ & Assessor \\
\hline
\end{tabular}


Table 1 Details of the intervention randomized controlled trials in which at least one arm involved wrist arthroscopy or shoulder arthroscopy (Continued)

\begin{tabular}{|c|c|c|c|c|c|c|c|c|c|c|c|c|}
\hline Burks 2009 & Australia & $\begin{array}{l}\text { Full-thickness } \\
\text { rotator cuff tear }\end{array}$ & $\begin{array}{l}\text { Arthroscopic single- } \\
\text { row rotator cuff } \\
\text { repair }\end{array}$ & 20 & 0 & $\begin{array}{l}\text { Arthroscopic double-row } \\
\text { rotator cuff repair }\end{array}$ & 20 & 0 & $\begin{array}{l}\text { Random } \\
\text { number } \\
\text { Generator; } \\
\text { Sealed } \\
\text { envelopes }\end{array}$ & At surgery & $\begin{array}{l}\text { UCLA, MRI, } \\
\text { Constant-Murley, } \\
\text { WORC, SANE, ASES, } \\
\text { ROM, strength }\end{array}$ & $\begin{array}{l}\text { Assessors } \\
\text { (radiologist and } \\
\text { examiner) }\end{array}$ \\
\hline Charron 2007 & USA & $\begin{array}{l}\text { Distal clavicle } \\
\text { osteolysis or post- } \\
\text { traumatic acromio- } \\
\text { clavicular arthrosis } \\
\text { without instability }\end{array}$ & $\begin{array}{l}\text { Arthroscopic distal } \\
\text { clavicle resection } \\
\text { with a direct } \\
\text { approach }\end{array}$ & 19 & 1 & $\begin{array}{l}\text { Arthroscopic distal clavicle } \\
\text { resection with an indirect } \\
\text { subacromial approach }\end{array}$ & 19 & 3 & $\begin{array}{l}\text { Order of } \\
\text { enrollment } \\
\text { (odd/even) }\end{array}$ & At enrollment & $\begin{array}{l}\text { ASES, ATH, time to } \\
\text { full return to sports }\end{array}$ & NR \\
\hline Chen 2010 & China & Frozen shoulder & $\begin{array}{l}\text { Arthroscopic release } \\
\text { of anterior capsular } \\
\text { structures }\end{array}$ & 42 & 1 & $\begin{array}{l}\text { Arthroscopic release } \\
\text { extended inferiorly and } \\
\text { posteriorly }\end{array}$ & 32 & 3 & $\begin{array}{l}\text { Computer; } \\
\text { Sealed } \\
\text { envelopes }\end{array}$ & At surgery & Constant, ROM & $\begin{array}{l}\text { Patients and } \\
\text { Assessors }\end{array}$ \\
\hline De Carli 2012 & Italy & $\begin{array}{l}\text { Idiopathic adhesive } \\
\text { shoulder capsulitis }\end{array}$ & $\begin{array}{l}\text { Arthroscopy } \\
\text { arthrolysis and } \\
\text { shoulder } \\
\text { manipulation }\end{array}$ & 25 & 2 & $\begin{array}{l}\text { Glenohumeral steroid } \\
\text { injections }\end{array}$ & 21 & 0 & NR & NR & $\begin{array}{l}\text { ROM, ASES, UCLA, } \\
\text { SST, Constant- } \\
\text { Murley }\end{array}$ & NR \\
\hline Dezaly 2011 & France & $\begin{array}{l}\text { Rotator cuff tear in } \\
\text { the over-60s }\end{array}$ & $\begin{array}{l}\text { Arthroscopic biceps } \\
\text { acromioplasty- } \\
\text { tenotomy and } \\
\text { repair }\end{array}$ & 71 & $3 ?^{+}$ & $\begin{array}{l}\text { Arthroscopic biceps } \\
\text { acromioplasty-tenotomy }\end{array}$ & 71 & $12 ?^{\dagger}$ & NR & $\begin{array}{l}\text { Day before } \\
\text { surgery }\end{array}$ & $\begin{array}{l}\text { Constant, } \\
\text { ultrasound tendon } \\
\text { healing }\end{array}$ & NR \\
\hline Elmlund 2009 & Sweden & $\begin{array}{l}\text { Recurrent shoulder } \\
\text { instability }\end{array}$ & $\begin{array}{l}\text { Arthroscopic } \\
\text { reconstruction with } \\
\text { polygluconate-B } \\
\text { polymer }\end{array}$ & 20 & 4 & $\begin{array}{l}\text { Arthroscopic } \\
\text { reconstruction with poly- } \\
\text { L-lactic acid polymer tack } \\
\text { implants }\end{array}$ & 20 & 3 & $\begin{array}{l}\text { Sealed } \\
\text { envelopes }\end{array}$ & $\begin{array}{l}\text { Just before } \\
\text { surgery }\end{array}$ & $\begin{array}{l}\text { Radiographs, CRP, } \\
\text { Constant, Rowe, } \\
\text { apprehension test, } \\
\text { strength, ROM, } \\
\text { recurrence of } \\
\text { instability }\end{array}$ & $\begin{array}{l}\text { Assessor } \\
\text { (radiologist) }\end{array}$ \\
\hline $\begin{array}{l}\text { Fabbriciani } \\
2004\end{array}$ & Italy & $\begin{array}{l}\text { Traumatic anterior } \\
\text { shoulder instability }\end{array}$ & Arthroscopic repair & 30 & NR & Open repair & 30 & NR & Computer & At surgery & Constant, Rowe & NR \\
\hline $\begin{array}{l}\text { Franceschi } \\
2008\end{array}$ & Italy & $\begin{array}{l}\text { Rotator cuff tear } \\
\text { and a type II SLAP } \\
\text { lesion in the over- } \\
50 \text { s }\end{array}$ & $\begin{array}{l}\text { Arthroscopic repair } \\
\text { of both lesions }\end{array}$ & 31 & 2 & $\begin{array}{l}\text { Arthroscopic rotator cuff } \\
\text { tear repair without repair } \\
\text { of the SLAP II lesion but } \\
\text { with tenotomy of the } \\
\text { long head of the biceps }\end{array}$ & 32 & 5 & $\begin{array}{l}\text { Random } \\
\text { number table; } \\
\text { Sealed } \\
\text { envelopes }\end{array}$ & At surgery & UCLA, ROM & NR \\
\hline $\begin{array}{l}\text { Freedman } \\
2007\end{array}$ & USA & $\begin{array}{l}\text { Refractory } \\
\text { acromioclavicular } \\
\text { joint pain }\end{array}$ & $\begin{array}{l}\text { Open distal clavicle } \\
\text { excision }\end{array}$ & 9 & 1 & $\begin{array}{l}\text { Arthroscopic distal clavicle } \\
\text { excision }\end{array}$ & 8 & 1 & NR & NR & $\begin{array}{l}\text { Pain VAS (primary). } \\
\text { modified ASES, } \\
\text { SF-36 }\end{array}$ & NR \\
\hline $\begin{array}{l}\text { Gartsman } \\
2004\end{array}$ & USA & $\begin{array}{l}\text { Full-thickness } \\
\text { rotator cuff tear }+ \\
\text { type } 2 \text { acromion }\end{array}$ & $\begin{array}{l}\text { Arthroscopic rotator } \\
\text { cuff repair + } \\
\text { subacromial } \\
\text { decompression }\end{array}$ & 47 & NR & $\begin{array}{l}\text { Arthroscopic rotator cuff } \\
\text { repair without } \\
\text { subacromial } \\
\text { decompression }\end{array}$ & 46 & NR & $\begin{array}{l}\text { Random } \\
\text { number table }\end{array}$ & At surgery & ASES & Patients \\
\hline Grasso 2009 & Italy & $\begin{array}{l}\text { Full-thickness } \\
\text { rotator cuff tear }\end{array}$ & $\begin{array}{l}\text { Arthroscopic single- } \\
\text { row rotator cuff } \\
\text { repair }\end{array}$ & 40 & 3 & $\begin{array}{l}\text { Arthroscopic double-row } \\
\text { rotator cuff repair }\end{array}$ & 40 & 5 & Computer & At surgery & $\begin{array}{l}\text { DASH, Constant, } \\
\text { strength }\end{array}$ & NR \\
\hline
\end{tabular}


Table 1 Details of the intervention randomized controlled trials in which at least one arm involved wrist arthroscopy or shoulder arthroscopy (Continued)

\begin{tabular}{|c|c|c|c|c|c|c|c|c|c|c|c|c|}
\hline Gumina 2012 & Italy & $\begin{array}{l}\text { Large full-thickness } \\
\text { posterosuperior } \\
\text { rotator cuff tear }\end{array}$ & $\begin{array}{l}\text { Arthroscopic repair } \\
\text { with platelet- } \\
\text { leukocyte } \\
\text { membrane }\end{array}$ & 40 & 1 & Arthroscopic repair & 40 & 3 & $\begin{array}{l}\text { Randomization } \\
\text { list; Sealed } \\
\text { envelopes }\end{array}$ & $\begin{array}{l}3 \text { days before } \\
\text { surgery }\end{array}$ & $\begin{array}{l}\text { Constant, MRI } \\
\text { (primary), SST }\end{array}$ & Assessors \\
\hline Haahr 2005 & Denmark & $\begin{array}{l}\text { Subacromial } \\
\text { impingement }\end{array}$ & $\begin{array}{l}\text { Arthroscopic } \\
\text { subacromial } \\
\text { decompression }\end{array}$ & 45 & 4 & Physiotherapy & 45 & 2 & $\begin{array}{l}\text { Computer; } \\
\text { Sealed } \\
\text { envelopes }\end{array}$ & NR & $\begin{array}{l}\text { Constant, pain VAS, } \\
\text { ROM, strength, ADL }\end{array}$ & NR \\
\hline Henkus 2009 & $\begin{array}{l}\text { Nether- } \\
\text { lands }\end{array}$ & $\begin{array}{l}\text { Primary } \\
\text { subacromial } \\
\text { impingement } \\
\text { without rotator } \\
\text { cuff rupture }\end{array}$ & $\begin{array}{l}\text { Arthroscopic } \\
\text { subacromial } \\
\text { bursectomy }\end{array}$ & 27 & 1 & $\begin{array}{l}\text { Debridement of } \\
\text { subacromial bursa + } \\
\text { arthroscopic } \\
\text { acromioplasty }\end{array}$ & 30 & 0 & $\begin{array}{l}\text { Automatically } \\
\text { generated } \\
\text { randomization } \\
\text { code }\end{array}$ & NR & $\begin{array}{l}\text { Constant, SST, pain } \\
\text { VAS, functional } \\
\text { impairment VAS }\end{array}$ & $\begin{array}{l}\text { Assessor and } \\
\text { group } 1 \\
\text { patients }\end{array}$ \\
\hline $\begin{array}{l}\text { Hiemstra } \\
2008\end{array}$ & Canada & Shoulder instability & Open stabilization & 24 & 0 & Arthroscopic stabilization & 24 & 0 & Computer & NR & $\begin{array}{l}\text { Strength (primary), } \\
\text { ASES, ROM }\end{array}$ & Assessor \\
\hline Husby 2003 & Norway & $\begin{array}{l}\text { Impingement } \\
\text { syndrome (Neer } \\
\text { grade II) }\end{array}$ & $\begin{array}{l}\text { Arthroscopic } \\
\text { subacromial } \\
\text { decompression }\end{array}$ & 20 & 5 & $\begin{array}{l}\text { Open subacromial } \\
\text { decompression }\end{array}$ & 19 & 0 & $\begin{array}{l}\text { Sealed } \\
\text { envelopes }\end{array}$ & At surgery & $\begin{array}{l}\text { UCLA, pain VAS, } \\
\text { satisfaction VAS, } \\
\text { strength, ROM }\end{array}$ & Assessor \\
\hline Kasten 2011 & Germany & $\begin{array}{l}\text { Supraspinatus } \\
\text { tendon rupture }\end{array}$ & Arthroscopic repair & 17 & 3 & Mini-open technique & 17 & 1 & $\begin{array}{l}\text { Order of } \\
\text { enrollment(first } \\
17 / \text { next 17) }\end{array}$ & NR & $\begin{array}{l}\text { NSAID use, pain, } \\
\text { Constant-Murley, } \\
\text { ASES, MRI }\end{array}$ & $\begin{array}{l}\text { Assessor } \\
\text { (radiologist) }\end{array}$ \\
\hline Ketola 2009 & Finland & $\begin{array}{l}\text { Shoulder } \\
\text { impingement } \\
\text { syndrome }\end{array}$ & Supervised exercise & 70 & 4 & $\begin{array}{l}\text { Arthroscopic } \\
\text { acromioplasty }+ \\
\text { supervised exercise }\end{array}$ & 70 & 2 & $\begin{array}{l}\text { Computer; } \\
\text { Sealed } \\
\text { envelopes }\end{array}$ & NR & $\begin{array}{l}\text { Pain VAS, ROM, } \\
\text { strength (primary), } \\
\text { cost-effectiveness }\end{array}$ & $\begin{array}{l}\text { Assessor } \\
\text { (physiotherapist) }\end{array}$ \\
\hline Kim 2011 & Korea & $\begin{array}{l}\text { Rotator cuff tear + } \\
\text { asymptomatic } \\
\text { acromioclavicular } \\
\text { arthritis }\end{array}$ & $\begin{array}{l}\text { Arthroscopic distal } \\
\text { clavicle resection } \\
\text { with rotator cuff } \\
\text { repair }\end{array}$ & 31 & 2 & $\begin{array}{l}\text { Arthroscopic rotator cuff } \\
\text { repair }\end{array}$ & 52 & 4 & $\begin{array}{l}\text { Random } \\
\text { number table }\end{array}$ & NR & $\begin{array}{l}\text { ASES, UCLA, pain, } \\
\text { AC joint tenderness, } \\
\text { cross body } \\
\text { adduction test }\end{array}$ & NR \\
\hline Kirkley 1999 & Canada & $\begin{array}{l}\text { First traumatic } \\
\text { anterior dislocation }\end{array}$ & $\begin{array}{l}\text { Immediate } \\
\text { arthroscopic } \\
\text { stabilization }\end{array}$ & 19 & 0 & $\begin{array}{l}\text { Immobilization and } \\
\text { rehabilitation }\end{array}$ & 21 & 2 & NR & NR & $\begin{array}{l}\text { WOSI, ROM, } \\
\text { redislocation }\end{array}$ & Assessor \\
\hline Koh 2011 & $\begin{array}{l}\text { South } \\
\text { Korea }\end{array}$ & Rotator cuff tear & $\begin{array}{l}\text { Arthroscopic single- } \\
\text { row repair }\end{array}$ & 37 & 6 & $\begin{array}{l}\text { Arthroscopic double-row } \\
\text { repair }\end{array}$ & 34 & 3 & Computer & At surgery & $\begin{array}{l}\text { Pain VAS, Constant, } \\
\text { ASES, UCLA, re-tear, } \\
\text { MRI }\end{array}$ & $\begin{array}{l}\text { Assessors } \\
\text { (radiologist and } \\
\text { examiner) }\end{array}$ \\
\hline Lindh 1993 & Sweden & $\begin{array}{l}\text { Shoulder } \\
\text { impingement }\end{array}$ & $\begin{array}{l}\text { Arthroscopic } \\
\text { subacromial } \\
\text { decompression }\end{array}$ & 10 & 0 & Open acromioplasty & 10 & 0 & NR & NR & $\begin{array}{l}\text { Osteophyte } \\
\text { recurrence, ROM, } \\
\text { UCLA }\end{array}$ & NR \\
\hline Ma 2012 & Taiwan & $\begin{array}{l}\text { Full-thickness } \\
\text { rotator cuff tear }\end{array}$ & $\begin{array}{l}\text { Arthroscopic single- } \\
\text { row repair }\end{array}$ & 32 & 5 & $\begin{array}{l}\text { Arthroscopic double-row } \\
\text { repair }\end{array}$ & 32 & 6 & $\begin{array}{l}\text { Computer; } \\
\text { Sealed } \\
\text { envelopes }\end{array}$ & At surgery & $\begin{array}{l}\text { UCLA, ASES, } \\
\text { strength, magnetic } \\
\text { resonance } \\
\text { arthrography }\end{array}$ & NR \\
\hline $\begin{array}{l}\text { MacDonald } \\
2011\end{array}$ & Canada & $\begin{array}{l}\text { Full-thickness } \\
\text { rotator cuff tear }\end{array}$ & $\begin{array}{l}\text { Arthroscopic repair } \\
+ \text { acromioplasty }\end{array}$ & 41 & 9 & Arthroscopic repair & 45 & 9 & $\begin{array}{l}\text { Computer; } \\
\text { Sealed } \\
\text { envelopes }\end{array}$ & At surgery & $\begin{array}{l}\text { WORC (primary), } \\
\text { ASES, revision }\end{array}$ & $\begin{array}{l}\text { Patients and } \\
\text { Assessor }\end{array}$ \\
\hline
\end{tabular}


Table 1 Details of the intervention randomized controlled trials in which at least one arm involved wrist arthroscopy or shoulder arthroscopy (Continued)

\begin{tabular}{|c|c|c|c|c|c|c|c|c|c|c|c|c|}
\hline $\begin{array}{l}\text { Magnusson } \\
2006\end{array}$ & Sweden & $\begin{array}{l}\text { Post-traumatic } \\
\text { shoulder instability }\end{array}$ & $\begin{array}{l}\text { Arthroscopic } \\
\text { Bankart } \\
\text { reconstruction with } \\
\text { polygluconate co- } \\
\text { polymer }\end{array}$ & 20 & 0 & $\begin{array}{l}\text { Arthroscopic Bankart } \\
\text { reconstruction with self- } \\
\text { reinforced poly-L-lactic } \\
\text { acid polymer }\end{array}$ & 20 & 0 & $\begin{array}{l}\text { Sealed } \\
\text { envelopes }\end{array}$ & $\begin{array}{l}\text { Just before } \\
\text { surgery }\end{array}$ & $\begin{array}{l}\text { Strength, ROM, } \\
\text { Rowe, Constant, } \\
\text { stability, } \\
\text { radiography }\end{array}$ & $\begin{array}{l}\text { Assessor } \\
\text { (radiologist) }\end{array}$ \\
\hline Milano 2007 & Italy & $\begin{array}{l}\text { Full-thickness } \\
\text { rotator cuff tear }\end{array}$ & $\begin{array}{l}\text { Arthroscopic repair } \\
+ \text { subacromial } \\
\text { decompression }\end{array}$ & 40 & 3 & Arthroscopic repair & 40 & 6 & Computer & At surgery & Constant, DASH & NR \\
\hline Milano 2010 & Italy & $\begin{array}{l}\text { Recurrent } \\
\text { traumatic anterior } \\
\text { shoulder instability }\end{array}$ & $\begin{array}{l}\text { Arthroscopic repair } \\
\text { with metal suture } \\
\text { anchor }\end{array}$ & 39 & 3 & $\begin{array}{l}\text { Arthroscopic repair with } \\
\text { biodegradable suture } \\
\text { anchor }\end{array}$ & 39 & 5 & $\begin{array}{l}\text { Random } \\
\text { sequence } \\
\text { generator; } \\
\text { Sealed } \\
\text { envelopes }\end{array}$ & At surgery & $\begin{array}{l}\text { DASH (primary),", } \\
\text { Rowe, Constant, } \\
\text { recurrence }\end{array}$ & Assessor \\
\hline Milano 2010 & Italy & $\begin{array}{l}\text { Full-thickness } \\
\text { rotator cuff tear }\end{array}$ & $\begin{array}{l}\text { Arthroscopic repair } \\
\text { with metal suture } \\
\text { anchor }\end{array}$ & 55 & 3 & $\begin{array}{l}\text { Arthroscopic repair with } \\
\text { biodegradable suture } \\
\text { anchor }\end{array}$ & 55 & 6 & $\begin{array}{l}\text { Random } \\
\text { sequence } \\
\text { generator }\end{array}$ & At surgery & DASH, Constant & Assessor \\
\hline Mohtadi 2008 & Canada & $\begin{array}{l}\text { Full-thickness } \\
\text { rotator cuff tear }\end{array}$ & Open repair & 37 & 8 & $\begin{array}{l}\text { Arthroscopic } \\
\text { acromioplasty with mini- } \\
\text { open repair }\end{array}$ & 36 & 5 & $\begin{array}{l}\text { Computer; } \\
\text { Sealed } \\
\text { envelopes }\end{array}$ & $N R$ & $\begin{array}{l}\text { RC-QOL (primary), } \\
\text { ASES, SRQ, FSET, } \\
\text { ROM, strength }\end{array}$ & Assessor \\
\hline $\begin{array}{l}\text { Monteiro } \\
2008\end{array}$ & Brazil & $\begin{array}{l}\text { Traumatic anterior } \\
\text { shoulder instability }\end{array}$ & $\begin{array}{l}\text { Arthroscopic repair } \\
\text { with anchors } \\
\text { loaded with } \\
\text { absorbable sutures }\end{array}$ & 25 & 4 & $\begin{array}{l}\text { Arthroscopic repair with } \\
\text { anchors loaded with } \\
\text { nonabsorbable sutures }\end{array}$ & 25 & 1 & $\begin{array}{l}\text { Sealed } \\
\text { envelopes }\end{array}$ & $N R$ & Rowe, ASOSS & Assessor \\
\hline Oh 2011 & $\begin{array}{l}\text { South } \\
\text { Korea }\end{array}$ & $\begin{array}{l}\text { Partial- or full- } \\
\text { thickness rotator } \\
\text { cuff tear }\end{array}$ & $\begin{array}{l}\text { Arthroscopic repair } \\
+\mathrm{HA} \\
\text { carboxymethylated } \\
\text { cellulose injection }\end{array}$ & 40 & NR & Arthroscopic repair & 40 & NR & Computer & NR & $\begin{array}{l}\text { Pain VAS, PROM, } \\
\text { ASES, } \\
\text { ultrasonography, } \\
\text { CTA }\end{array}$ & $\begin{array}{l}\text { Injection and } \\
\text { Assessor }\end{array}$ \\
\hline Randelli 2011 & Italy & $\begin{array}{l}\text { Complete rotator } \\
\text { cuff tear }\end{array}$ & $\begin{array}{l}\text { Arthroscopic repair } \\
+ \text { autologous } \\
\text { platelet rich plasma }\end{array}$ & 26 & 4 & Arthroscopic repair & 27 & 4 & $\begin{array}{l}\text { Computer; } \\
\text { Sealed } \\
\text { envelopes }\end{array}$ & At surgery & $\begin{array}{l}\text { Pain VAS, SST, } \\
\text { UCLA, Constant, } \\
\text { strength, MRI }\end{array}$ & $\begin{array}{l}\text { Assessors } \\
\text { (radiologist and } \\
\text { examiner) }\end{array}$ \\
\hline $\begin{array}{l}\text { Robinson } \\
2008\end{array}$ & UK & $\begin{array}{l}\text { First-time } \\
\text { traumatic anterior } \\
\text { dislocation }\end{array}$ & $\begin{array}{l}\text { Arthroscopic } \\
\text { examination and } \\
\text { lavage }\end{array}$ & 45 & 3 & $\begin{array}{l}\text { Arthroscopic examination } \\
\text { and Bankart lesion repair }\end{array}$ & 43 & 1 & $\begin{array}{l}\text { Computer; } \\
\text { weighted } \\
\text { minimization }\end{array}$ & $N R$ & $\begin{array}{l}\text { Recurrence, } \\
\text { functional scores, } \\
\text { DASH, patient } \\
\text { satisfaction, SF-36, } \\
\text { WOSI, ROM, cost }\end{array}$ & $\begin{array}{l}\text { Patients and } \\
\text { Assessor } \\
\text { (physiotherapist) }\end{array}$ \\
\hline Rodeo 2012 & USA & $\begin{array}{l}\text { Full-thickness } \\
\text { rotator cuff tear }\end{array}$ & $\begin{array}{l}\text { Arthroscopic repair } \\
+ \text { platelet-rich fibrin } \\
\text { matrix }\end{array}$ & 40 & 5 & Arthroscopic repair & 39 & 7 & $\begin{array}{l}\text { Sealed } \\
\text { envelopes }\end{array}$ & At surgery & $\begin{array}{l}\text { Healing on } \\
\text { ultrasound } \\
\text { (primary), ASES, } \\
\text { L'Insalata, manual } \\
\text { muscle testing }\end{array}$ & $\begin{array}{l}\text { Patients and } \\
\text { Assessor }\end{array}$ \\
\hline Sachs 1994 & USA & $\begin{array}{l}\text { Impingement } \\
\text { syndrome (stage II) }\end{array}$ & $\begin{array}{l}\text { Arthroscopic } \\
\text { acromioplasty }\end{array}$ & 22 & 3 & Open acromioplasty & 22 & 0 & NR & $N R$ & $\begin{array}{l}\text { Pain, function, ROM, } \\
\text { strength, RTA, LOHS }\end{array}$ & NR \\
\hline Shin 2012 & $\begin{array}{l}\text { South } \\
\text { Korea }\end{array}$ & $\begin{array}{l}\text { Small-medium } \\
\text { rotator cuff tear }\end{array}$ & $\begin{array}{l}\text { Arthroscopic repair } \\
+ \text { acromioplasty }\end{array}$ & 75 & 15 & Arthroscopic repair & 75 & 15 & NR & Before surgery & $\begin{array}{l}\text { VAS, UCLA, ASES, } \\
\text { Constant, MRI, ROM }\end{array}$ & NR \\
\hline
\end{tabular}


Table 1 Details of the intervention randomized controlled trials in which at least one arm involved wrist arthroscopy or shoulder arthroscopy (Continued)

\begin{tabular}{|c|c|c|c|c|c|c|c|c|c|c|c|c|}
\hline Shin 2012 & $\begin{array}{l}\text { South } \\
\text { Korea }\end{array}$ & $\begin{array}{l}\text { Partial-thickness } \\
\text { articular-sided rota- } \\
\text { tor cuff tear }\end{array}$ & $\begin{array}{l}\text { Arthroscopic repair } \\
\text { with transtendon } \\
\text { technique }\end{array}$ & 24 & 0 & $\begin{array}{l}\text { Arthroscopic repair with } \\
\text { full-thickness conversion }\end{array}$ & 24 & 0 & Computer & At surgery & $\begin{array}{l}\text { Pain and } \\
\text { satisfaction VAS, } \\
\text { ASES, Constant, MRI, } \\
\text { ROM }\end{array}$ & $\begin{array}{l}\text { Assessors } \\
\text { (radiologist and } \\
\text { examiner) }\end{array}$ \\
\hline $\begin{array}{l}\text { Silberberg } \\
2011\end{array}$ & Spain & $\begin{array}{l}\text { Isolated type II } \\
\text { SLAP lesion }\end{array}$ & $\begin{array}{l}\text { Arthroscopic repair } \\
\text { with vertical suture }\end{array}$ & 15 & 0 & $\begin{array}{l}\text { Arthroscopic repair with } \\
\text { horizontal suture }\end{array}$ & 17 & 0 & Minimization & At surgery & $\begin{array}{l}\text { Pain and instability } \\
\text { VAS, ASES, ROM }\end{array}$ & Assessor \\
\hline $\begin{array}{l}\text { Spangehl } \\
2002\end{array}$ & Canada & $\begin{array}{l}\text { Impingement } \\
\text { syndrome }\end{array}$ & $\begin{array}{l}\text { Arthroscopic } \\
\text { acromioplasty }\end{array}$ & 32 & $? / 25^{\dagger}$ & Open acromioplasty & 30 & $? / 25^{+}$ & $N R$ & NR & $\begin{array}{l}\text { Pain and function } \\
\text { VAS (primary), } \\
\text { UCLA, satisfaction, } \\
\text { strength }\end{array}$ & Assessor \\
\hline Sperber 2001 & Sweden & $\begin{array}{l}\text { Traumatic anterior } \\
\text { shoulder instability }\end{array}$ & $\begin{array}{l}\text { Arthroscopic } \\
\text { stabilization }\end{array}$ & 30 & NR & Open stabilization & 26 & NR & $\begin{array}{l}\text { Sealed } \\
\text { envelopes }\end{array}$ & At surgery & $\begin{array}{l}\text { Recurrence, ROM, } \\
\text { apprehension sign, } \\
\text { relocation test, } \\
\text { Constant, Rowe }\end{array}$ & NR \\
\hline Syed 2010 & USA & $\begin{array}{l}\text { Soft tissue fluid } \\
\text { retention after } \\
\text { shoulder } \\
\text { arthroscopy }\end{array}$ & $\begin{array}{l}\text { Fenestrated outflow } \\
\text { cannula }\end{array}$ & 14 & 0 & Conventional cannula & 14 & 0 & $\begin{array}{l}\text { Sealed } \\
\text { envelopes }\end{array}$ & NR & Fluid weight gain & Patients \\
\hline Tan 2006 & UK & $\begin{array}{l}\text { Recurrent } \\
\text { traumatic anterior } \\
\text { instability }\end{array}$ & $\begin{array}{l}\text { Arthroscopic } \\
\text { Bankart repair with } \\
\text { nonabsorbable } \\
\text { anchor }\end{array}$ & 65 & 2 & $\begin{array}{l}\text { Arthroscopic Bankart } \\
\text { repair with absorbable } \\
\text { anchor }\end{array}$ & 65 & 4 & $\begin{array}{l}\text { Sealed } \\
\text { envelopes }\end{array}$ & At surgery & $\begin{array}{l}\text { OISS, pain and } \\
\text { instability VAS, } \\
\text { SF-12, recurrence }\end{array}$ & $\begin{array}{l}\text { Patients and } \\
\text { Assessors }\end{array}$ \\
\hline Taverna 2007 & Italy & $\begin{array}{l}\text { Chronic } \\
\text { supraspinatus } \\
\text { tendinosis }\end{array}$ & $\begin{array}{l}\text { Arthroscopic } \\
\text { subacromial } \\
\text { decompression }\end{array}$ & 30 & 0 & $\begin{array}{l}\text { Radiofrequency-based } \\
\text { plasma microtenotomy }\end{array}$ & 30 & 0 & $\begin{array}{l}\text { Sealed } \\
\text { envelopes }\end{array}$ & $\begin{array}{l}\text { Just before } \\
\text { surgery }\end{array}$ & $\begin{array}{l}\text { Pain VAS, Constant, } \\
\text { ASES, UCLA, SF-36 }\end{array}$ & $\begin{array}{l}\text { Patients and } \\
\text { Assessor } \\
\text { (physician) }\end{array}$ \\
\hline Wintzell 1996 & Sweden & $\begin{array}{l}\text { Acute traumatic } \\
\text { primary anterior } \\
\text { dislocation }\end{array}$ & Arthroscopic lavage & 15 & 0 & Conservative treatment & 15 & 0 & $N R$ & NR & $\begin{array}{l}\text { Recurrenc, } \\
\text { apprehension test, } \\
\text { ROM, Lysholm score }\end{array}$ & Assessor \\
\hline
\end{tabular}

*The references are listed in Additional file 2.

${ }^{+}$Dropouts/withdrawals were mentioned but the exact number in each group was not clear in the article.

Abbreviations in alphabetical order:

ADL Activities of Daily Living, ASES American Shoulder and Elbow Surgeons shoulder score, ASOSS Athletic Shoulder Outcome Scoring System, ATH Athletic Shoulder Scoring System score, Constant Constant shoulder score, CRP C-Reactive Protein, CTA Computed Tomography Arthrography, DASH Disabilities of the Arm, Shoulder and Hand score, D/W dropouts/withdrawals, FSET Functional Shoulder Elevation Test, LOHS length of hospital stay, MRI Magnetic Resonance Imaging, $N$ number of patients randomized, NR not reported, OISS Oxford Instability Shoulder Score, PROM Passive Range Of Motion, RC-QOL Rotator Cuff Quality Of Life score, ROM Range Of Motion, Rowe Rowe shoulder score, RTA return to activities, RTW return to work, SANE Single Assessment Numeric Evaluation score, SF-12 Short Form 12 survey, SF-36 Short Form 36 survey, SRQ Shoulder Rating Questionnaire, SST Simple Shoulder Test, UCLA University of California-Los Angeles shoulder rating scale, VAS Visual Analogue Scale, WORC Western Ontario Rotator Cuff index, WOSI Western Ontario Shoulder Instability index. 
The median modified Jadad score for the wrist arthroscopy intervention RCTs was 0.5 (range 0-1) and for the shoulder arthroscopy intervention RCTs was 3.0 (range 0-5). The quality of the shoulder RCTs was significantly higher than that for the wrist RCTs $(p=0.012)$.

\section{Discussion}

Our study shows that despite the increasing use of wrist arthroscopy in the treatment of various wrist disorders the efficacy of arthroscopically performed interventions has only been studied in 4 quasi-randomized studies. This can be compared to 50 randomized or quasirandomized studies of significantly higher quality for arthroscopically performed shoulder interventions, yet both procedures were first described in the literature in the late 1970s.

Since their introduction as diagnostic tools, both wrist and shoulder arthroscopy have undergone technical advancement and broader clinical applications. However, they appear to diverge in the extent to which they have been evaluated scientifically. It might be argued that shoulder disorders are more common and therefore it would be easier to conduct randomized trials. However, wrist arthroscopy is being used for several wrist disorders that are relatively common. Besides, multicenter trials can be conducted when a condition is not that common to allow enrollment of an adequate number of patients in a reasonable time. In contrast to wrist arthroscopy, endoscopic carpal tunnel release, an arthroscopic procedure, first described in the literature in the late 1980s, has been evaluated in numerous intervention RCTs, including a number of high quality trials as judged by the Cochrane reviews [10]. Also, our review of shoulder arthroscopy RCTs shows that it is possible to conduct good-quality surgical intervention trials involving arthroscopy.

Arthroscopic interventions are now used for new areas in upper extremity surgery such as thumb carpometacarpal osteoarthritis, a common condition, still without evidence from randomized studies. Because conducting good-quality surgical RCTs, with the many factors involved, is generally more difficult than pharmaceutical trials, proposals have been presented recently to facilitate surgical trials $[11,12]$. The lack of high-level evidence, based on good-quality randomized trials, to support the large number of surgical interventions performed through wrist arthroscopy should be a concern not only to health care payers and providers but also to patients.

Like other quality assessment systems, the Jadad scale has its limitations. Although the scale considers the appropriateness of the randomization method, which is fundamental, it does not include concealment. We have however extracted the data concerning concealment for each trial, when such data were reported (Table 1).
Further, blinding of patients may not be feasible in surgical interventions. However, we also considered blinding of outcome assessors and this should be feasible in surgical trials. Another limitation is the possible existence of RCTs that the search did not capture. However, we do not believe that the search missed any eligible wrist intervention RCTs.

It is highly unlikely that a study that had used blinding or achieved complete follow-up with no drop-outs or withdrawals would not report these in the published article as important strengths. We considered studies that only mentioned using "sealed envelopes" without specifying how the randomization sequence was generated ( 2 wrist studies and 11 shoulder studies) as not having reported the randomization method and thus were not awarded any points for randomization. Even if we assume that these studies had used appropriate methods in generating the randomization sequence the results would be similar (median score 1.5 vs 3.0; $\mathrm{p}=0.041$ ).

In our search we could not find any previous studies that have assessed the quality of intervention trials involving wrist arthroscopy. With regard to RCTs that involved shoulder arthroscopy, there have been systematic reviews of intervention trials for specific shoulder disorders that included interventions done through arthroscopy. Most of these reviews used different quality scales and therefore could not be compared directly with our study. For example, a systematic review of interventions for anterior shoulder instability assessed the quality of 3 trials with a 12-item scale that included concealment and blinding (each item scored 0,1 or 2 for a best possible total score of 24 points) giving them a score of 17,16 and 15 , respectively [13]. The modified Jadad score for the same 3 trials in our study was 3, 2 and 0 , respectively, which reflects the fact that the modified Jadad scale focuses on the unambiguous reporting of the fundamental issues of randomization, blinding and drop-outs/withdrawals.

In one previous systematic review that used the original Jadad scale in assessing the quality of 54 rotator cuff RCTs published from 2001 to 2011, the mean Jadad score was 3.0 [14]. The authors concluded that most trials were of high quality $(66 \%$ had a Jadad score $>3.0)$ but because almost two-thirds of the high-quality studies were nonoperative trials they suggested that the rotator cuff literature lacks high quality RCTs that are relevant to surgical clinical practice [14]. In another report based on the "comparative effectiveness of nonoperative and operative treatments for rotator cuff tears" systematic review of literature from 1990 to 2009, the authors concluded that the "RCT literature was of particularly low quality with high risk of bias from the manner in which the studies had been conducted" [15]. Thus, despite our finding that most intervention RCTs involving shoulder arthroscopy were of significantly higher quality than the 
very few wrist arthroscopy trials that have been performed, there is need for further improved shoulder surgical RCTs. For example, six RCTs (published since 2002) that have assessed the efficacy of knee arthroscopy in the treatment of osteoarthritis [16] are probably of substantially higher quality than most shoulder arthroscopy RCTs.

In a study that estimated the number of upper extremity ambulatory procedures performed in the United States in 2006, including wrist and shoulder arthroscopic interventions, the authors concluded that the resources utilized by these procedures are substantial and suggested that evidence-based clinical indications and outcomes of many of these upper extremity procedures remain poorly defined [3]. For interventions involving wrist arthroscopy, our systematic review shows that there is currently a lack of good evidence supporting the efficacy of these procedures.

\section{Conclusions}

This systematic review revealed that the efficacy of arthroscopically performed wrist interventions has been studied in only 4 quasi-randomized studies compared to 50 randomized or quasi-randomized studies of significantly higher quality assessing interventions performed through shoulder arthroscopy. In order to advance evidence-based care of patients with wrist disorders, there is a need for high-quality RCTs designed to assess the efficacy of the procedures currently performed through wrist arthroscopy.

\section{Additional files}

Additional file 1: Search details for randomized controlled trials (RCTs) involving wrist arthroscopy or shoulder arthroscopy.

Additional file 2: The randomized controlled trials in which at least one arm involved intervention performed through wrist arthroscopy or shoulder arthroscopy, included in the systematic review.

\section{Competing interests}

The authors of this manuscript declare that they have no financial or non-financial competing interests.

\section{Authors' contributions}

IA and RSR designed the study. RSR performed the search. KT, RSR and IA performed the review. KT and IA performed the statistical analysis. KT and IA prepared the initial draft of the manuscript. All authors read and approved the final version.

\section{Author details}

'Department of Orthopedics, Hässleholm Hospital, Hässleholm SE-28125, Sweden. ${ }^{2}$ Unit for Hand \& Microsurgery, GECOT, La Laguna, Tenerife, Spain. ${ }^{3}$ Department of Clinical Sciences, Lund University, Lund, Sweden.

Received: 26 September 2013 Accepted: 1 July 2014

Published: 25 July 2014

References

1. Wolf JM, Dukas A, Pensak M: Advances in wrist arthroscopy. J Am Acad Orthop Surg 2012, 20:725-734.

2. Slutsky DJ: Current innovations in wrist arthroscopy. J Hand Surg Am 2012, 37:1932-1941.

3. Jain NB, Higgins LD, Losina E, Collins J, Blazar PE, Katz JN: Epidemiology of musculoskeletal upper extremity ambulatory surgery in the United States. BMC Musculoskelet Disord 2014, 15:4.

4. Adla DN, Rowsell M, Pandey R: Cost-effectiveness of open versus arthroscopic rotator cuff repair. J Shoulder Elbow Surg 2010, 19:258-261.

5. Ahsan ZS, Yao J: Complications of wrist arthroscopy. Arthroscopy 2012, 28:855-859.

6. Schulz KF, Altman DG, Moher D: CONSORT 2010 statement: updated guidelines for reporting parallel group randomised trials. BMJ 2010, 340:c332.

7. Liberati A, Altman DG, Tetzlaff J, Mulrow C, Gotzsche PC, loannidis JP, Clarke M, Devereaux PJ, Kleijnen J, Moher D: The PRISMA statement for reporting systematic reviews and meta-analyses of studies that evaluate health care interventions: explanation and elaboration. Ann Intern Med 2009, 151:W65-W94.

8. Jadad AR, Moore RA, Carroll D, Jenkinson C, Reynolds DJ, Gavaghan DJ, McQuay HJ: Assessing the quality of reports of randomized clinical trials: is blinding necessary? Control Clin Trials 1996, 17:1-12.

9. Gummesson C, Atroshi I, Ekdahl C: The quality of reporting and outcome measures in randomized clinical trials related to upper-extremity disorders. J Hand Surg Am 2004, 29:727-734.

10. Scholten RJ, Mink van der Molen A, Uitdehaag BM, Bouter LM, de Vet HC: Surgical treatment options for carpal tunnel syndrome. Cochrane Database Syst Rev 2007, 4:CD003905. doi:10.1002/14651858.CD003905.pub3.

11. McCulloch P, Cook JA, Altman DG, Heneghan C, Diener MK: IDEAL framework for surgical innovation 1: the idea and development stages. BMJ 2013, 346:f3012

12. Ergina PL, Barkun JS, McCulloch P, Cook JA, Altman DG: IDEAL framework for surgical innovation 2: observational studies in the exploration and assessment stages. BMJ 2013, 346:f3011.

13. Pulavarti RS, Symes TH, Rangan A: Surgical interventions for anterior shoulder instability in adults. Cochrane Database Syst Rev 2009, 4: CD005077. doi:10.1002/14651858.CD005077.pub2.

14. McCormick F, Cvetanovich GL, Kim JM, Harris JD, Gupta AK, Abrams GD, Romeo AA, Provencher MT: An assessment of the quality of rotator cuff randomized controlled trials: utilizing the Jadad score and CONSORT criteria. J Shoulder Elbow Surg 2013, 22:1180-1185.

15. Butler M, Forte M, Braman J, Swiontkowski M, Kane RL: Nonoperative and operative treatments for rotator cuff tears: future research needs. Identification of future research needs from comparative effectiveness review No. 22 [Internet]. Rockville (MD): Agency for Healthcare Research and Quality (US); 2013. (Future Research Needs Papers, No. 39.) Available from: http://www. ncbi.nlm.nih.gov/books/NBK153178/.

16. Katz JN, Brownlee SA, Jones MH: The role of arthroscopy in the management of knee osteoarthritis. Best Pract Res Clin Rheumatol 2014 28:143-156

doi:10.1186/1471-2474-15-252

Cite this article as: Tadjerbashi et al:: Intervention randomized controlled trials involving wrist and shoulder arthroscopy: a systematic review. BMC Musculoskeletal Disorders 2014 15:252.

\section{Acknowledgements}

This research was supported and partially funded by Hässleholm Hospital.

The funder had no role in the design, the collection, analysis and interpretation of data, the writing of the manuscript or the decision to submit the manuscript for publication. 